\section{Beyond the diffraction-limit biological imaging by saturated excitation microscopy}

\author{
Masahito Yamanaka, ${ }^{a}$ Shogo Kawano, ${ }^{a}$ \\ Katsumasa Fujita, ${ }^{\mathrm{b}, *}$ Nicholas I. Smith, and \\ Satoshi Kawata \\ a Osaka University, Department of Frontier Biosciences, 2-1 \\ Yamadaoka, Suita, Osaka 565-871, Japan \\ ${ }^{b}$ Osaka University, Department of Applied Physics, 2-1 \\ Yamadaoka, Suita, Osaka, 565-0871, Japan \\ ${ }^{\mathrm{C}}$ Osaka University, Department of Applied Physics, 2-1 \\ Yamadaoka, Suita, Osaka, 565-0871, Japan and RIKEN, \\ Nanophotonics Laboratory, Hirosawa, Wako, Saitama \\ 351-0198 Japan
}

\begin{abstract}
We demonstrate high-resolution fluorescence imaging in biological samples by saturated excitation (SAX) microscopy. In this technique, we saturate the population of fluorescence molecules at the excited state with high excitation intensity to induce strong nonlinear fluorescence responses in the center of laser focus, which contributes the improvement of the spatial resolution in three dimensions. Using SAX microscopy, we observed stained microtubules in HeLa cells with improved spatial resolution. We also measured the relation of the fluorescence and excitation intensity with several kinds of fluorescence dyes and, in the results, confirmed that SAX microscopy has the potential to observe any kind of fluorescence samples in current usage. ๑ 2008 Society of PhotoOptical Instrumentation Engineers. [DOI: 10.1117/1.2992595]
\end{abstract}

Keywords: high-resolution; fluorescence microscopy; confocal microscopy; saturated excitation; biological imaging.

Paper 08201LR received Jul. 24, 2008; revised manuscript received Aug. 14, 2008; accepted for publication Aug. 18, 2008; published online Óct. 9, 2008; corrected Nov. 18, 2008.

\section{Introduction}

In recent developments in fluorescence microscopy, the diffraction barrier in the spatial resolution has been broken and the resolution has been reduced to $<50 \mathrm{~nm}$. High-resolution imaging techniques have achieved nanoscale resolution by precise control of fluorescence emission with stimulated emission, ${ }^{1,2}$ The combination of structured illumination and saturation of fluorescence molecules at the excited state, ${ }^{3-5}$ and statistic and stochastic location techniques with photoswitchable fluorescence molecules. ${ }^{6-9}$

In this paper, we present an alternative method which may be more easily generalized to observe any type of fluorescence samples in current usage. In the saturated excitation (SAX) microscopy technique, ${ }^{10}$ the spatial resolution is improved in three dimensions using strong nonlinear fluores-

Address all correspondence to: Katsumasa Fujita, Department of Applied Physics, Osaka University, 2-1 Yamadoaka, Suita, Osaka, 565-0871, Japan. Email: fujita@ap.eng.osaka-u.ac.jp

*Tel: +816-6879-7847; E-mail: fujita@ap.eng.osaka-u.ac.jp cence responses induced by saturation of fluorescence molecules at the excited state with high excitation intensity, which can be realized by adding laser-intensity modulators and a lock-in amplifier to a typical confocal fluorescence microscope. Because the saturation effect is inherent to any fluorescence molecule, SAX microscopy can realize high-resolution imaging in various applications using fluorescence probes. Here, we demonstrate the observation of biological samples using SAX microscopy and present fluorescence responses of dye molecules under saturated excitation, which show the capability of high-resolution imaging by SAX microscopy with dyes in current usage.

\section{Principle of SAX Microscopy}

In SAX microscopy, ${ }^{10}$ improvement in spatial resolution is achieved by exploiting the nonlinearity in the fluorescence signals, which is induced when the target fluorescence molecule excited states become saturated due to the excited-state lifetime and the limited number of fluorescence molecules in the focal volume of the laser light. The saturation phenomena appear most prominently in the center of the laser spot, where the relationship between the excitation intensity and fluorescence signals shows strong nonlinearity. The spatial dependence of the nonlinear fluorescence responses can then be used for the improvement of spatial resolution to give theoretically unlimited improvement of the spatial resolution in three dimensions. To detect the nonlinear fluorescence signals induced by saturated excitation, we implemented the use of temporal modulation of the excitation intensity at a fundamental frequency $(\omega)$ and consequent demodulation of the fluorescence intensity at higher-order harmonic frequencies $(2 \omega, 3 \omega, \ldots) .{ }^{10}$ As the excitation intensity is temporally modulated and saturated excitation occurs, the modulated fluorescence signals are distorted because of the nonlinear relation of the excitation and the fluorescence intensity. As a result, the distorted signal includes higher-order harmonic frequency $(2 \omega, 3 \omega, \ldots)$ components as well as the fundamental frequency $(\omega)$. Thus, the harmonic demodulation of fluorescence signals allows us to extract the nonlinear fluorescence responses induced by saturated excitation at the center of the excitation point spread function (PSF) and gives fluorescence signals from a region smaller than the PSF to improve the spatial resolution in three dimensions. An optical system for SAX microscopy can be realized by adding laser intensity modulators and a lock-in amplifier to a typical confocal fluorescence microscope.

\section{High-Resolution Fluorescence Images of Microtubules in HeLa Cells}

We observed microtubles stained with ATTO488 in a fixed HeLa cell to confirm the improvement of spatial resolution in SAX microscopy. Figures 1(a) and 1(b) show the fluorescence images of the sample obtained by SAX microscopy and conventional confocal fluorescence microscopy, respectively. The intensity profiles spanning the dotted lines in these images are also shown. In the intensity profile in the image by SAX microscopy, several peaks, which were not observed in the image by conventional confocal microscopy, became clearly

1083-3668/2008/13(5)/050507/3/\$25.00 @ 2008 SPIE 


\section{JBO LETTERS}
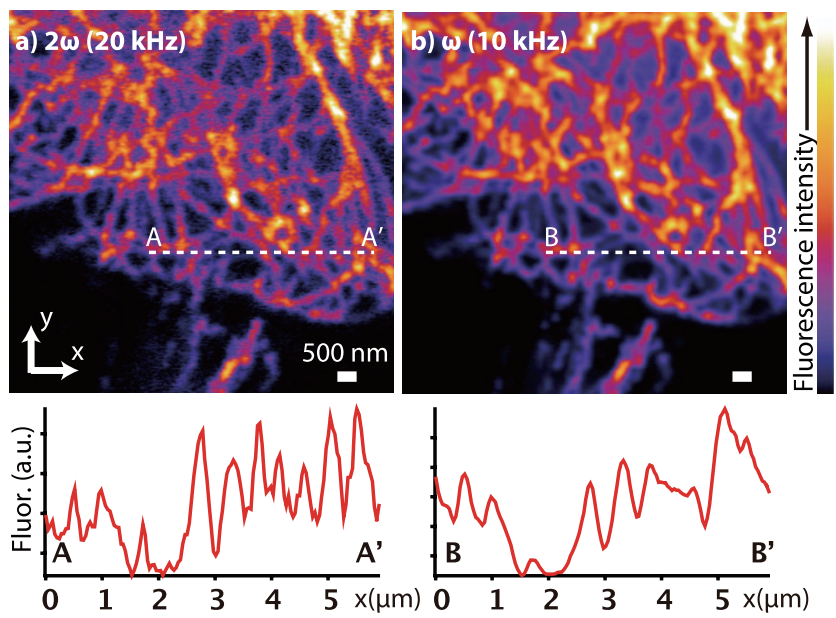

Fig. 1 Fluorescence images of microtubules in HeLa cells in the focal plane $(x-y$ image). The fluorescence images were obtained with demodulation at (a) the second harmonic frequency and (b) the fundamental frequency. The plot at the bottom of the images shows the intensity profiles of the dotted lines in the images.

observable. In addition, compared to peaks in the intensity profiles, the width of peaks in SAX microscopy were $\sim 1.4$ times smaller than those in conventional confocal imaging. From these results, we confirmed the improvement of spatial resolution by SAX microscopy in fluorescence observation of biological samples. In the experiments, the fluorescence dye was excited with continuous-wave laser light at a wavelength of $488 \mathrm{~nm}$ and an oil-immersion objective lens with a numerical aperture (NA) of 1.4. We modulated the excitation intensity at $10 \mathrm{kHz}$ and demodulated the fluorescence intensity at the fundamental frequency $(10 \mathrm{kHz})$ and the second harmonic frequency $(20 \mathrm{kHz})$. For $10 \mathrm{kHz}$ (fundamental frequency) demodulation, we chose the excitation intensity as $2 \mathrm{~kW} / \mathrm{cm}^{2}$. This intensity is weak enough so as not to induce saturated excitation or harmonic distortion, giving the same spatial resolution that would be obtained by conventional confocal fluorescence microscopy. For SAX microscopy, the $20 \mathrm{kHz}$ (second harmonic) demodulation frequency was used with an excitation intensity of $66 \mathrm{~kW} / \mathrm{cm}^{2}$, which provided saturated excitation at the center of the laser spot. The images were not averaged and were taken with a pixel dwell time of $0.3 \mathrm{~ms}$, at $256 \times 256$ pixels, resulting in the image acquisition time of $20 \mathrm{~s}$.

Figure 2 shows the SAX fluorescence imaging performance along the optical axis ( $x-z$ image) and intensity profiles of the microtubule-stained HeLa cell. Compared with the intensity profiles, more peaks became clearly observable in the result by SAX microscopy, while each peak also became $\sim 1.25$ times narrower than in the result of conventional confocal imaging. From this result, we confirmed that SAX microscopy can be used to improve the spatial resolution in the $z$ direction as well as lateral directions. In this experiment, the image size was $256 \times 128$ pixels and an image was recorded in $\sim 10 \mathrm{~s}$. Other experimental conditions were same as that for Fig. 1.

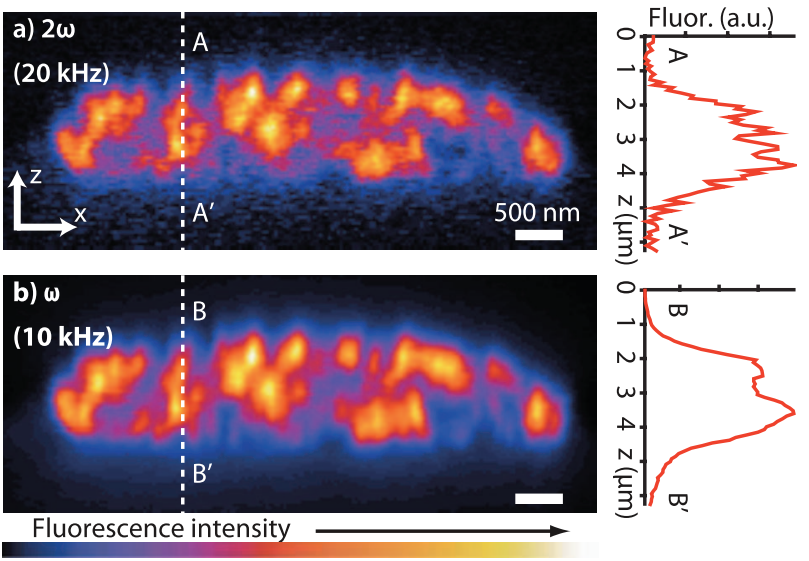

Fig. 2 Fluorescence images of microtubules in HeLa cells along the optical axis ( $x-z$ image). The fluorescence images were obtained with demodulation at (a) the second harmonic frequency and (b) the fundamental frequency. The plot on the right of the images shows the intensity profiles of the dotted lines in the images.

\section{Nonlinear Fluorescence Responses of Dyes}

We also note that SAX microscopy can be used with a wide range of dyes without regard to spectral limitations. This can be seen by the harmonic demodulation and successful extraction of nonlinear fluorescence signals from a range of fluorescent dyes (Fig. 3). We measured the demodulated fluorescence intensity from ATTO488, Rhodamine 6G, and Rhodamine B solution with a concentration of $10 \mu \mathrm{M}$ and Alexa fluor 488 goat antimouse $\mathrm{IgG}$ solution. The fluorescence solution was excited with a water-immersion objective lens $(\mathrm{NA}=1.2)$. We modulated the excitation intensity at a frequency of $10 \mathrm{kHz}$ and demodulated the fluorescence signals at the fundamental frequency $\omega(10 \mathrm{kHz})$ and also at the harmonic frequencies $2 \omega(20 \mathrm{kHz}), 3 \omega(30 \mathrm{kHz}), 4 \omega(40 \mathrm{kHz})$, and $5 \omega(50 \mathrm{kHz})$. In Fig. 3, the fluorescence intensity given at the $n$th $(n$ $=1,2,3, \ldots)$ order harmonic frequency was proportional to the $n$ 'th power of the excitation intensity and shows that the third or even higher harmonics retain a close-to-ideal nonlinear dependence on the excitation intensity. It also shows that the harmonic signals are also saturated at high excitation intensities associated with increase of higher-frequency components. This confirmation of successful harmonic demodulation of fluorescence in a range of dyes is promising for the application of the technique to high-resolution fluorescence microscopy without specific limitations on the dye.

\section{Discussion}

In this letter, we confirmed that SAX microscopy improves the 3-D spatial resolution beyond the diffraction limit in the condition of biological observation using a simpler optical system and imaging scheme compared with other techniques for high-resolution fluorescence microscopy. The improvement of the spatial resolution in SAX microscopy is achieved by exploiting higher-order nonlinear responses in fluorescence emission in the same manner as in multiphoton excitation fluorescence microscopy. However, the spatial resolution given by multiphoton excitation fluorescence microscopy is almost the same as that of single-photon excitation confocal fluorescence microscopy, because multiphoton excitation re- 


\section{JBO LETTERS}
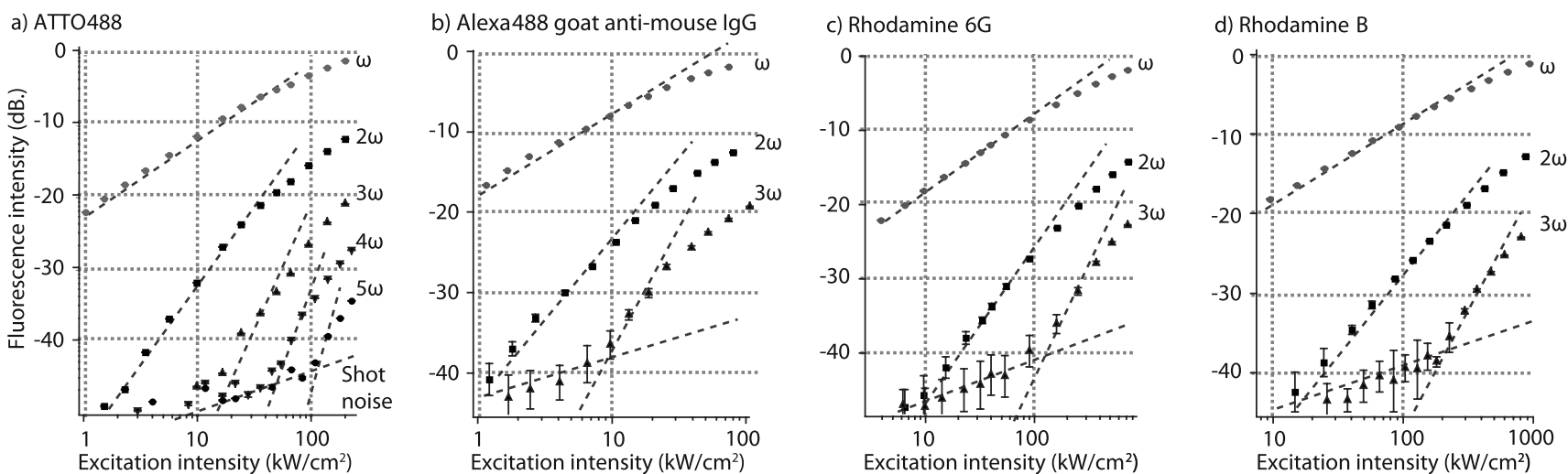

Fig. 3 Demodulated fluorescence intensity measured with various excitation intensities from (a) ATTO488, (b) Alexa fluor 488 goat antimouse IgG, (c) Rhodamine $6 \mathrm{G}$, and (d) Rhodamine B. Excitation wavelengths were $488 \mathrm{~nm}$ for (a,b) and $532 \mathrm{~nm}$ for (c,d). Modulation frequency of the excitation intensity was $\omega(10 \mathrm{kHz})$. Dotted lines in (a) show slopes of $1,2,3,4,5$, and 0.5 from above and of 1, 2, 3, and 0.5 in (b-d). The shot noise is seen as the signal increasing with the slope of 0.5 . The shot noise is the main source of the noise in the detection of nonlinear fluorescence signals and disturbed the detection of fluorescence signals given at higher-order harmonic frequencies.

quires a longer wavelength to excite molecules with multiple low-energy photons. On the other hand, SAX microscopy uses higher-order nonlinearity without an increase of excitation wavelength, which substantially improves the spatial resolution of fluorescence microscopy beyond the diffraction limit.

Although the requirement of strong excitation intensity gives rise to more photobleaching effects in SAX microscopy than in conventional fluorescence microscopy, this paper demonstrates that SAX microscopy is applicable to imaging of biological samples. To reduce the photobleaching effect, fluorescence excitation by temporally separated pulsed laser instead is effective, as shown in Ref. 11. In observations of living biological samples, the higher excitation intensity may exhibit not only photobleaching but also other undesirable effects, such as defunctionalization of proteins by a large temperature rise or reactive oxygen species induced via photobleaching of fluorescence molecules as used in chromophore-assisted laser inactivation of proteins, ${ }^{12,13}$ both of which inhibit cellular functions and may make observations of various in vivo phenomena difficult.

The ability of SAX microscopy to observe biological samples with no spectral limitation of the dye is a significant advance in high-resolution fluorescence microscopy, because the technique can be applied in a wide range of microscopic observations performed with fluorescence probes in current usage. Our technique is expected to make great contributions to the scientific investigations in biology and medicine if the obstacles described above are overcome and further improvement of the spatial resolution is achieved experimentally.

\section{Acknowledgment}

This study was supported by the Industrial Technology Research Grant Program in 2006 from the New Energy and Industrial Technology Development Organization (NEDO) in Japan.

\section{References}

1. S. W. Hell and J. Wichmann, "Breaking the diffraction resolution limit by stimulated emission:stimulated-emission-depletion fluorescence microscopy," Opt. Lett. 19, 780-782 (1994).

2. V. Westphal, S. O. Rizzoli, M. A. Lauterbach, D. Kamin, R. Jahn, and S. W. Hell, "Video-rate far-field nanoscopy dissects synaptic vesicles movement," Science 320, 246-249 (2008).

3. M. G. Gustafsson, "Nonlinear structured-illuminatio microscopy:wide-field fluorescence imaging with theoretically unlimited resolution," Proc. Natl. Acad. Sci. U.S.A. 102, 13081-13086 (2005).

4. R. Heintzmann and T. M. Jovin, "Saturated patterned excitation microscopy-A concept for optical resolution improvement," J. Opt. Soc. Am. A 19, 1599-1609 (2002).

5. R. Heinzmann, "Saturated patterned excitation microscopy with twodimensional excitation patterns," Micron 34, 283-291 (2003).

6. E. Betzig, G. H. Patterson, R. Sougrat, O. W. Lindwasser, S. Olenych, J. S. Bonifacino, M. W. Davidson, J. Lippincott-Schwartz, and H. F. Hess, "Imaging intracellular fluorescent proteins at nanometer resolution," Science 313, 1642-1645 (2006).

7. H. Shroff, C. G. Galbraith, J. A. Galbraith, H. White, J. Gillette, S. Olenych, M. W. Davidson, and E. Betzig, "Dual-color superresolution imaging of genetically expressed probes within individual adhesion complexes," Proc. Natl. Acad. Sci. U.S.A. 104, 20308-20313 (2008).

8. M. J. Rust, M. Bates, and X. Zhauang, "Sub-diffraction-limit imaging by stochastic optical reconstruction microscopy (STORM)," Nat. Methods 3, 793-796 (2006).

9. B. Huang, W. Wang, M. Bates, and X. Zhuang, "Three-dimensional super-resolution imaging by stochastic optical reconstruction microscopy," Science 319, 810-813 (2008).

10. K. Fujita, M. Kobayashi, S. Kawano, M. Yamanaka, and S. Kawata, "High-resolution confocal microscopy by saturated excitation of fluorescence," Phys. Rev. Lett. 99, 228105 (2007).

11. G. Donnert, C. Eggeling, and S. W. Hell, "Major signal increase in fluorescence microscopy through dark-state relaxation," Nat. Methods 4, 81-86 (2007).

12. D. G. Jay, "Selective destruction of protein function by chromophoreassisted laser inactivation," Proc. Natl. Acad. Sci. U.S.A. 85, 54545458 (1988).

13. T. Tanabe, M. Oyamada, K. Fujita, P. Dai, H. Tanaka, and T. Takamatsu, "Multiphoton excitation-evoked chromophore-assisted laser inactivation using green fluorescent protein," Nat. Methods 2, 503505 (2005). 